\title{
CUSTOM HIP ARTHROPLASTY
}

\author{
Kalin Mihov, Maksim Zagorov, Svetoslav Dobrilov, Atanas Tabakov, Gergana Nenova \\ Department of Orthopedics and Traumatology, UMHAT St. Marina, Varna
}

\begin{abstract}
Total hip replacement is a rapidly growing procedure due to pain relief, restoring the range of motion and patient's satisfaction. The primary goal is to restore the individual geometry of the patient's hip joint, to achieve long-term component survival and most importantly - to improve the patient's quality of life.

In past decades this surgery has had several limitations such as patient's age, bone morphology (incl. anatomical deformities), previous surgeries, etc. Recently, with the development of modern implants (cups and stems) these limitations have been eliminated.

Young patients indicated for THA are always a great challenge, because of their functional requirements, life expectancy, anatomical variations (due to congenital or acquired disorders), greater mobility and higher risk of aseptic loosening.

Standard cementless stems have some unsolved issues such as fixed intra/extramedullary dimensions, proximal stress shielding, impingement, etc. They are based on 2D planning and often have a mismatch between the acetabular and the femoral center of rotation.

Custom femoral stems are based on a specific 3D scan of the hip joint, which presents the individual shape of the acetabulum and especially that of the femoral canal. This allows for optimal bone support for the stem, preserving bone substance, excellent bone-stem contact and most importantly - restores the center of rotation.

For the period 2010-2014 we have operated on 16 patients, 8 were with osteoarthrosis $(\mathrm{OA})$; 4 - with avascular necrosis (AVN); 2 - with dysplastic hips (DDH) and two - with posttraumatic osteoarthrosis. The followup is in 6-42 months.

We performed THA with a modified posterior surgical approach with minimal femoral reaming, due to individual femoral rasp with the same size as the customized femoral stem.

During the follow-up period we found no complications. The Harris Hip Score was $97 \mathrm{pts}$. and $85 \%$ of the patients had regular physical exercises for 3 weeks.
\end{abstract}

Keywords: osteoarthrosis, THA, cementless stems, custom hip, 3D-planning

Address for correspondence:

Kalin Mihov M.D.

1 Hristo Smirnenski Blvd

Dept. of Orthopedics and Traumatology

University Hospital "St. Marina"

Varna 9010, Bulgaria

e-mail: svetlyo_d@yahoo.com

Received: May 30, 2016

Accepted: July 22, 2016

\section{INTRODUCTION}

Total hip and total knee arthroplasty are rapidly growing procedures. In the USA, growth expectancy is $600 \%$ till the year 2030 . The reasons for this are many, but probably the most important one is excellent functional results after appropriate treatment. Nowadays, THA allows restoration of the individual geometry of the patient, minimal wear and longterm fixation of the bone component. For the pa- 
tient this means better quality of life - maximizing the range of motion without pain and greater physical activity.

In the recent years, with the development of better components, performing THA in younger patients becomes possible. The challenges in such patients are higher functional requirements and increased life expectancy. These patients often present with anatomical hip deformities due to dysplasia, posttraumatic arthrosis and recent surgical treatment. Younger patients are predisposed to aseptic loosening due to micro movement and incomplete "fit and fill" $(3,5,6)$.

Cementless stems have some disadvantages, particularly when applied in such patients. These stems have fixed extra/intramedullary dimension, which can't be used in certain cases with altered anatomy. There is a high risk of proximal stress shielding, impingement and some disadvantages related to modular neck corrosion, fracture or high level of metal ions.

Primary stem stability is a prerequisite to bone ingrowth and long-term fixation. Stability depends on the filling of the proximal femur and the anatomical position of the stem (fit) $(7,8)$. Primary OA and dysplastic hips are correlated with great anatomical variations of the intramedullary canal and femoral neck (anteversion, offset, femoral neck angle) $(6,7)$.

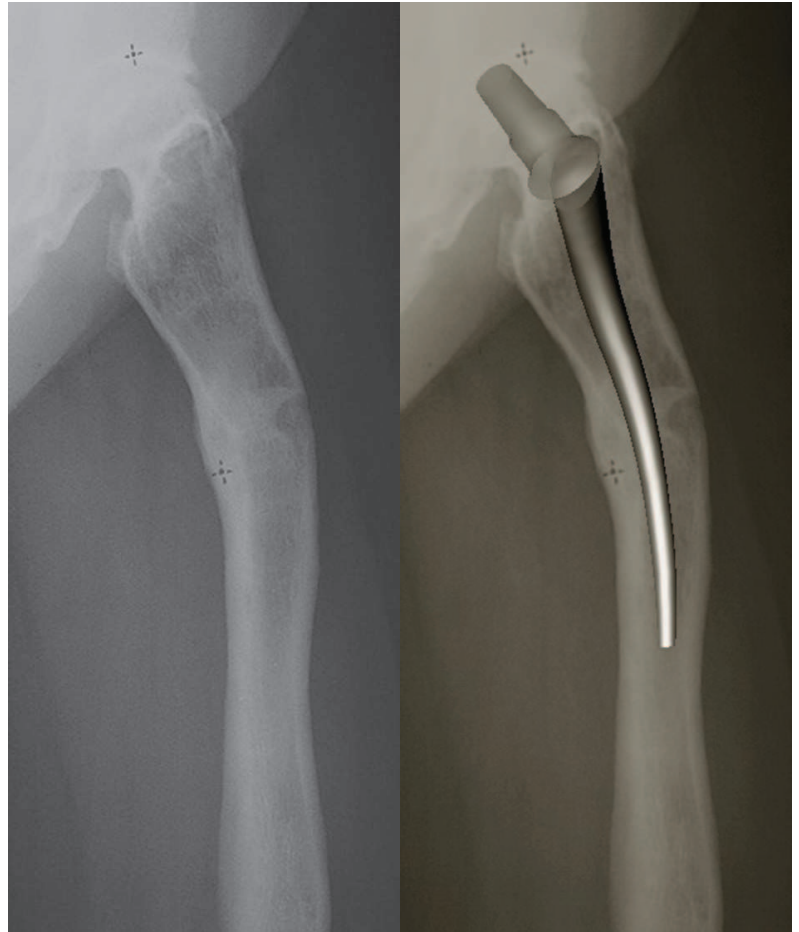

Fig. 1. Proximal femur deformity (left) and superimposed custom stem (right)

There are great differences in the tridimensional anatomy of the femoral canal, as well as in the extramedullary parameters, between dysplastic hips and primary OA $(7,8)$. The stage of dysplasia is correlated with the difficulties in the restoration of the acetabular center of rotation, which is necessary for the
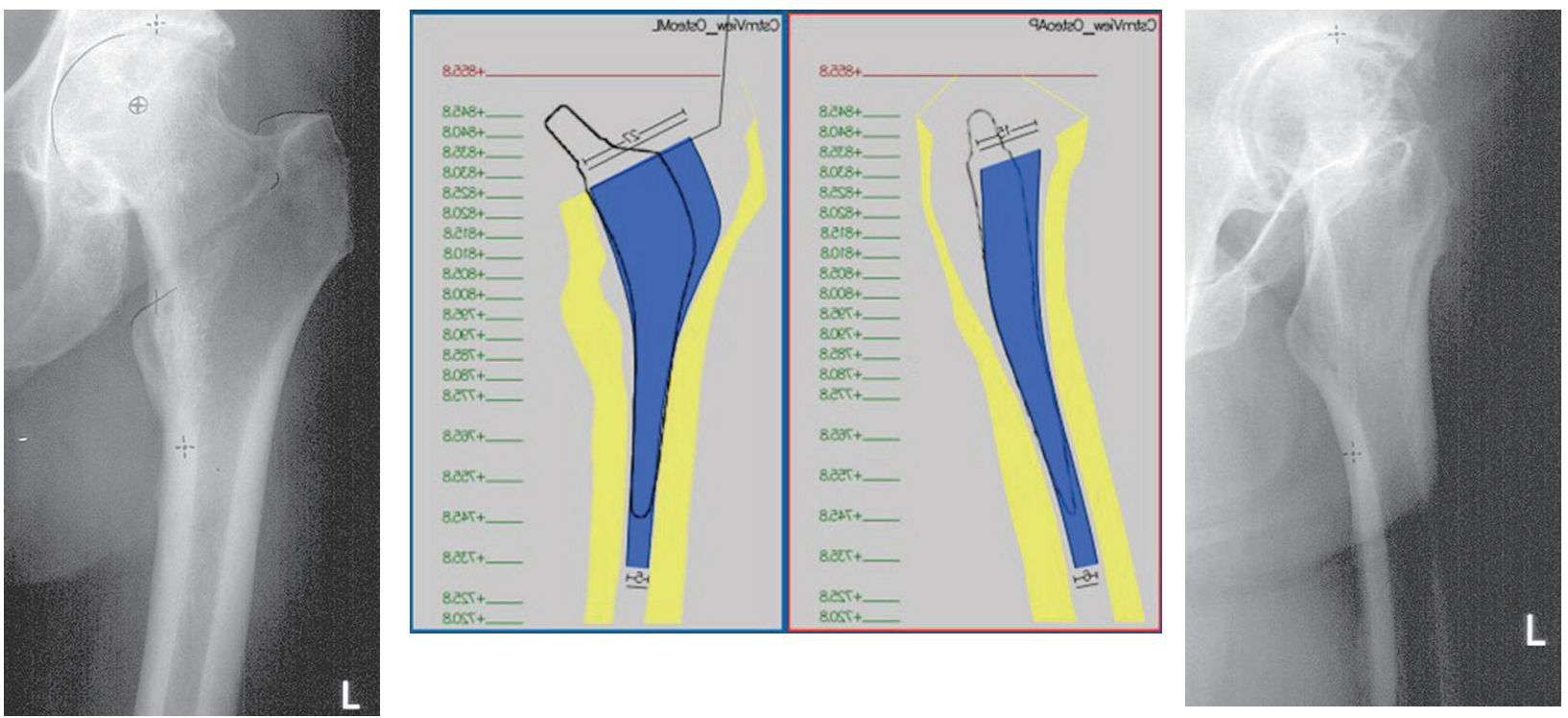

Fig. 2. Increased canal flare index 

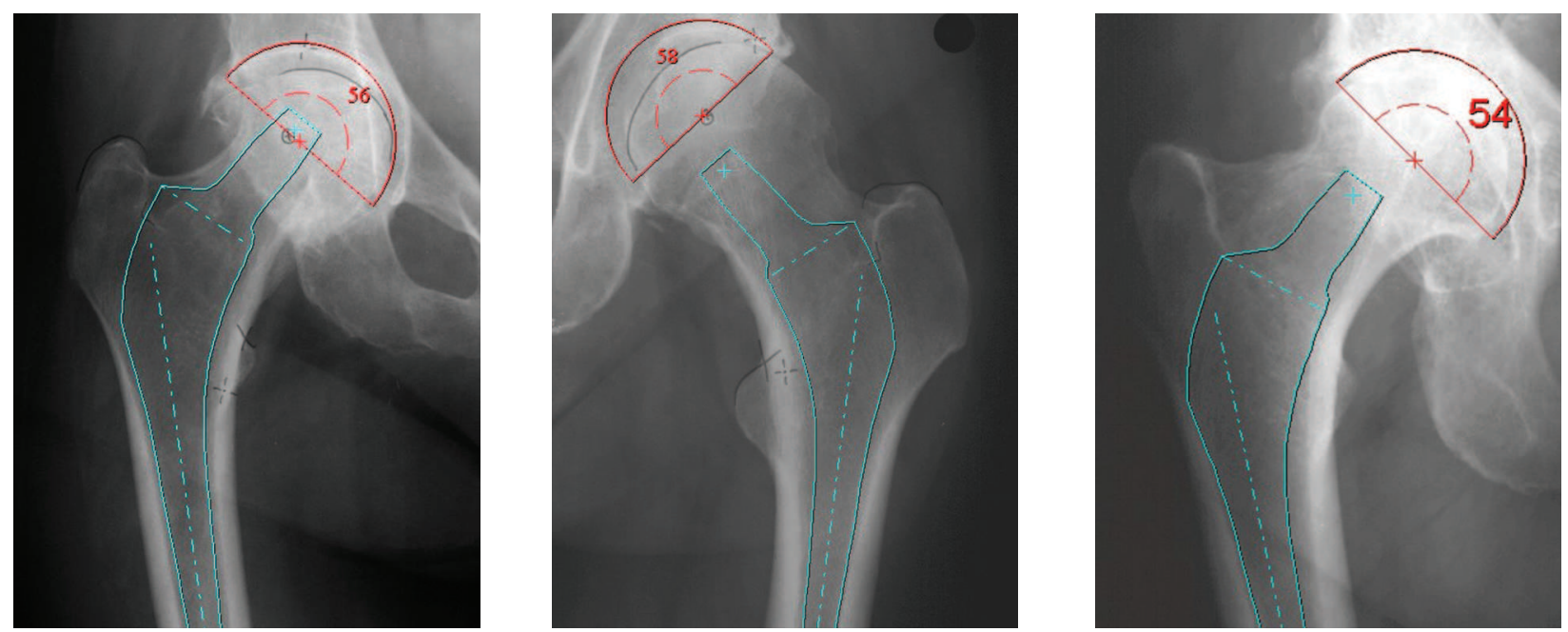

Fig. 3. Standard cementless stem (left picture) in a good intramedullary position and optimal reconstruction of the center of rotation (COR); admissible intramedullary position (in the middle), but indicated for longer valgus neck; good intramedullary position (right picture), but indicated for longer varus neck

correct joint kinematics and the lever of the abductor musculature $(10,11)$.

Difficulties in the points of intramedullary variations are: femur deformities (Fig. 1), increased canal flare index (Fig. 2), lateral curvature, and femur helitorsion.

The extramedullary variations are in the acetabular/femoral offset, the femoral neck length and anteversion, and the femoral neck angle (FNA). All these parameters define the lever of the abductor musculature.

Generally, the femoral offset was measured on AP radiographs (2D image) without considering anteversion and external rotation, leading to measurement errors thereof. These differences in the measurements can be found in up to 40 percent of cases $(6,16)$. According to Asayama et al. (1) clinical manifestation of the abductor weakness presents when the femoral offset decreases more than $12 \%(5 \mathrm{~mm})$ and clinically manifests as decreased over $28 \%$.

The standard stems are very well adapted intramedullary, but sometimes there is a mismatch between the center of rotation of the stem and the center of rotation of the femoral head of the patient (Fig. $3)$. Very often the femoral stems, designed for optimal proximal filling don't fit optimally in the metaphysical zone (12).

Apart from the intramedullary canal, the abnormal femoral anatomy is demonstrated also in the extramedullary parameters of the proximal femur (9).

When the position of the stem (the "fit and fill") is not optimal and the center of rotation is not restored, the most important indications for a successful hip replacement are not performed $(1,2)$. Such cases are indicated for customized hip prosthesis.

\section{MATERIALS AND METHODS}

From 2010 till 2014, 16 patients were operated on in the Department of Orthopedics and Traumatology, 14 men and 2 women, 8 of them suffering from osteoarthrosis, 4 - from avascular necrosis of the femoral head, two had dysplasia of the hip and two had post-traumatic osteoarthrosis. The average age was $54(20-69)$, and the average weight was $86.5 \mathrm{~kg}$ (BMI - 32.4). The postoperative follow-up period was between 6 and 42 months.

We took into account several factors during the patient selection:

$\diamond$ The Noble index;

$\diamond$ Conventional 2D planning (MediCAD) (Fig. 4);

$\diamond$ 3D CT planning (Symbios protocol) (Fig. 5);

The CT protocol is based on $5 \mathrm{~mm}$ cuts from the acetabulum to the greater trochanter, and $10 \mathrm{~mm}$ cuts from the lesser trochanter to the femoral isthmus. This allows precise intramedullary reconstruction of the femur. The anteversion is calculated with horizontal cuts on the level of the 2nd metatarsal, femoral condyles and $10 \mathrm{~mm}$ above the lesser trochanter. 

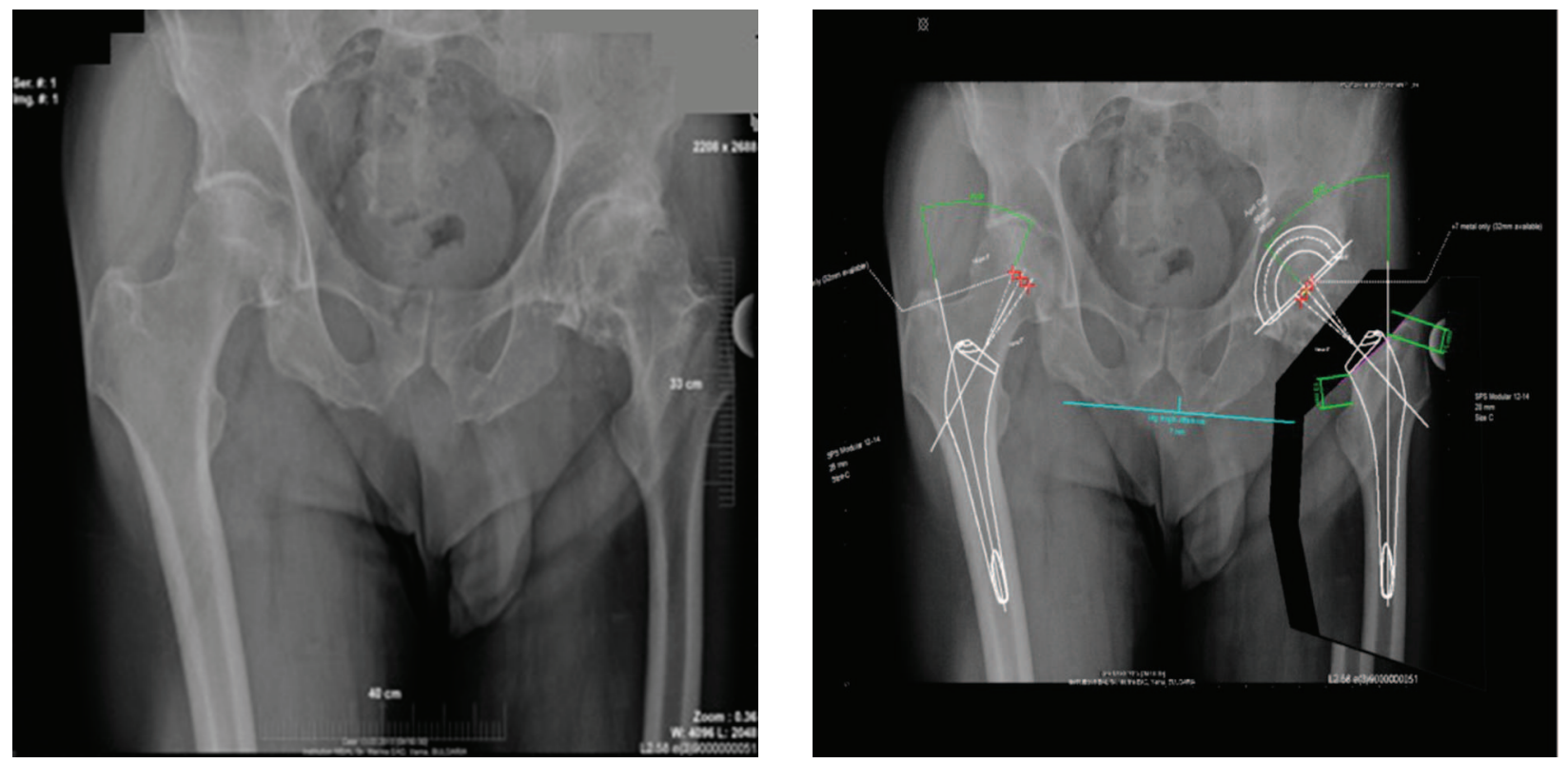

Fig. 4. Conventional 2 D planning (MediCAD)

AP acetabular dimension is formed with additional cuts. This protocol makes possible the calculation of the femoral neck angle (FNA) and the femoral anteversion (Fig. 5) (4).

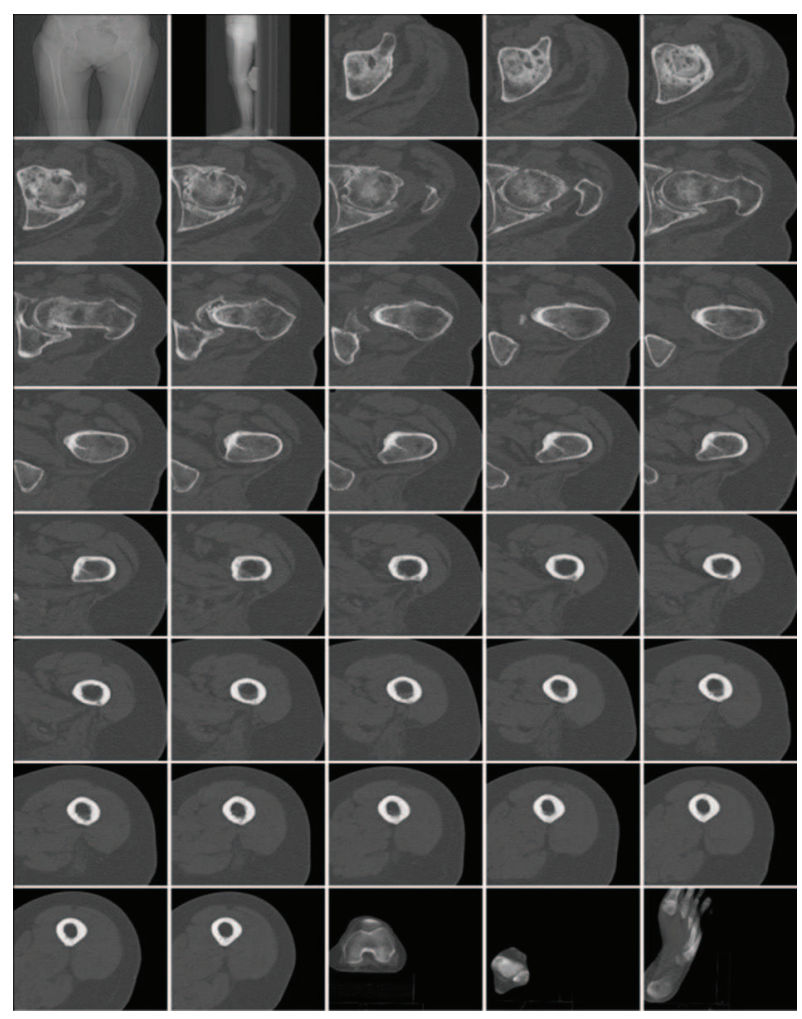

Fig. 5. CT protocol for 3D planning of the individual femoral stem
Custom stem design is based on the preoperative X-ray images and the CT protocol of each patient. 3D reconstruction of the femoral canal is necessary to avoid overreaming of the spongious bone, which results in cortical contact of the stem. The metaphyseal spongious bone is impacted, before stem implantation, with custom rasp designed as the stem. This CT protocol makes possible the correction of extramedullary parameters - offset and neck anteversion (9).

Preoperatively, we use actual size planning (1:1), available in the surgery room - the so-called "Face-Osteo" plan (Fig. 6). It shows bone resection parameters needed for precise stem implantation (values are projected over an X-ray image) and CT level calibration.

The following distances were measured:

1. (A) - from the lesser trochanter, the level of the osteotomy on the medial neck;

2. (B) - from the lesser trochanter to the medial top of the femoral neck;

3. (C) - from the lateral stem curve to the greater trochanter;

4. (D) - from the femoral axis to the top of the femoral head;

5. (F) - from the lateral stem curve to the top of the femoral head 


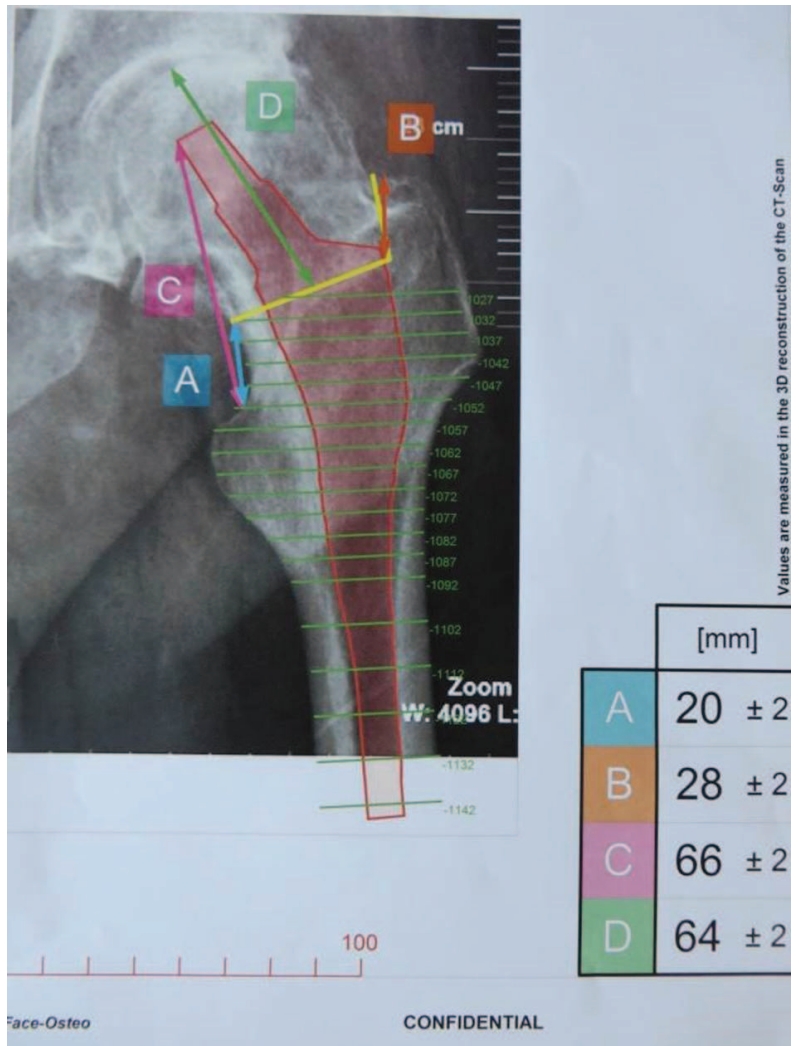

Fig. 6. „Face-Osteo“ planning

Additional measurements:

6. (E) - from the calcar resection to the top of the femoral head

7. (G) - the size of the femoral neck.

Another important component is CT reconstruction of the bone density in the metaphysealdiaphyseal area with the stem in situ (Fig. 7) which allows:

$\diamond$ Color contrast assessment of the density of the cancellous bone (black, green, yellow and red);

$\diamond$ Visualization of the areas where bone mass removal is necessary for the correct stroke of the stem;

$\Delta$ Control of the correct position of the stem at the level of the osteotomy (medio-lateral and antero-posterior);

The next stage of preoperative planning is to determine the anteversion of the stem. Fig. 8 shows the angular geometry of the femoral stem, the knee, and the foot, and we can see:

$\diamond$ Gait angle

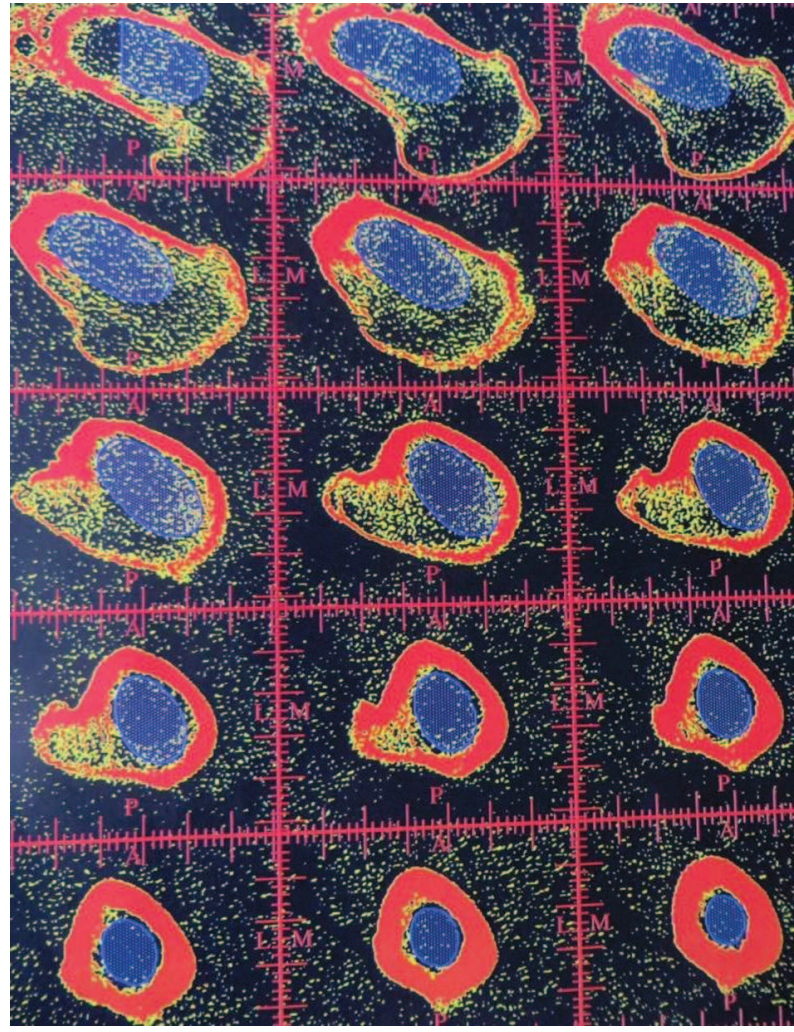

Fig. 7. CT reconstruction of the bone density

$\diamond$ helitorsion of the stem

$\diamond$ extramedullar angle need for correction, which shows the final anteversion according to the bicondylar line (PBCP).

The standard targeted anteversion is $15^{\circ}$, if there are no additional instructions from the surgeon.

Other additional plannings which we use are those for cup position, femur position before and after prosthesis reposition.

"Cup planning" is based again on CT reconstruction in axial, sagittal and coronal plan over the cup center of rotation (Fig. 9). It allows the calculatiion of the level of acetabular reaming for optimal cup position (coverage, version, lateralisation) and preserving enough bone substance.

While planning for our patients we received the following data:

$\diamond$ anteversion angle of the femur (A): average $15.4^{\circ}\left(10^{\circ}-20^{\circ}\right)$

$\diamond$ helitorsion $(\mathrm{H})$ : average $-17^{\circ}\left(2^{\circ}-39^{\circ}\right)$;

$\diamond$ delta $(\Delta)$ angle $(\mathrm{A}-\mathrm{H})$ : from $-19^{\circ}$ to $+18^{\circ}$;

$\diamond$ acetabular inclination: average $-52^{\circ}\left(49^{\circ}-57^{\circ}\right)$; 


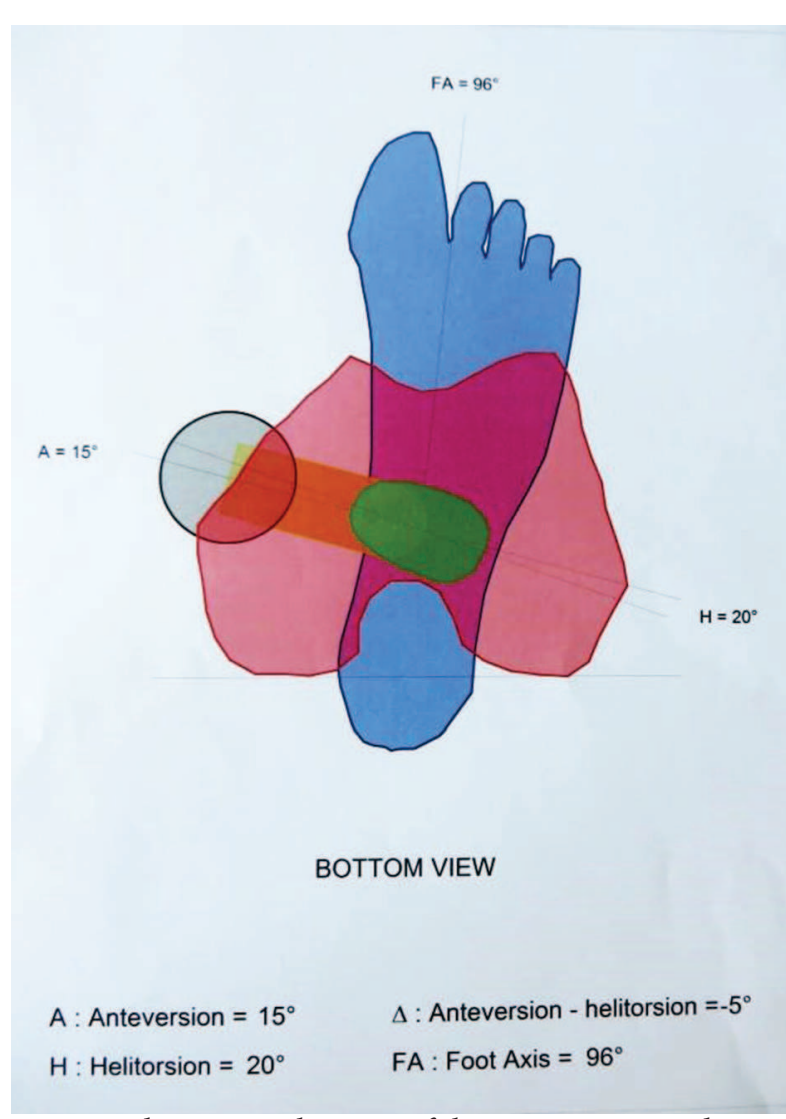

Fig. 8. Adjustment planning of the stem corresponding to the lower limb anatomy

$\diamond$ acetabular anteversion: average $-18^{\circ}\left(12^{\circ}-24^{\circ}\right)$.

Planned cup inclination was $45^{\circ}$ and $20^{\circ}$ anteversion. Bone resections were calculated as follows:

$\diamond(\mathrm{A})-17 \mathrm{~mm}(11 \mathrm{~mm}-21 \mathrm{~mm})$

$\diamond(B)-37 m m(20 m m-67 m m)$

$\diamond(C)-38 \mathrm{~mm}(27 \mathrm{~mm}-67 \mathrm{~mm})$

$\diamond(\mathrm{D})-52 \mathrm{~mm}(45 \mathrm{~mm}-64 \mathrm{~mm})$

$\diamond(\mathrm{F})-66 \mathrm{~mm}$ (available only in 3 patients)

We performed the standard posterior surgical approach with preserving $\mathrm{m}$. piriformis. The metaphyseal spongious bone was impacted with a smooth impactor, excluding 4 patients who needed tooth (aggressive) rasp (Fig. 10). We implanted titanium HA cups Hillock/MaxiMom (Symbios, Yverdon, Switzerland) and crosslinked polyethylene insert/ MOM. The customized stem is with 2/3 hydroxyapatite coverage (HA) (Symbios, Yverdon, Switzerland) and variable femoral heads: ceramic $-28.32 \mathrm{~mm}$ Alumina and $36 \mathrm{~mm}$ Delta; $32.36 \mathrm{~mm}$ Cr Co, MaxiMom (Fig. 6, 7).
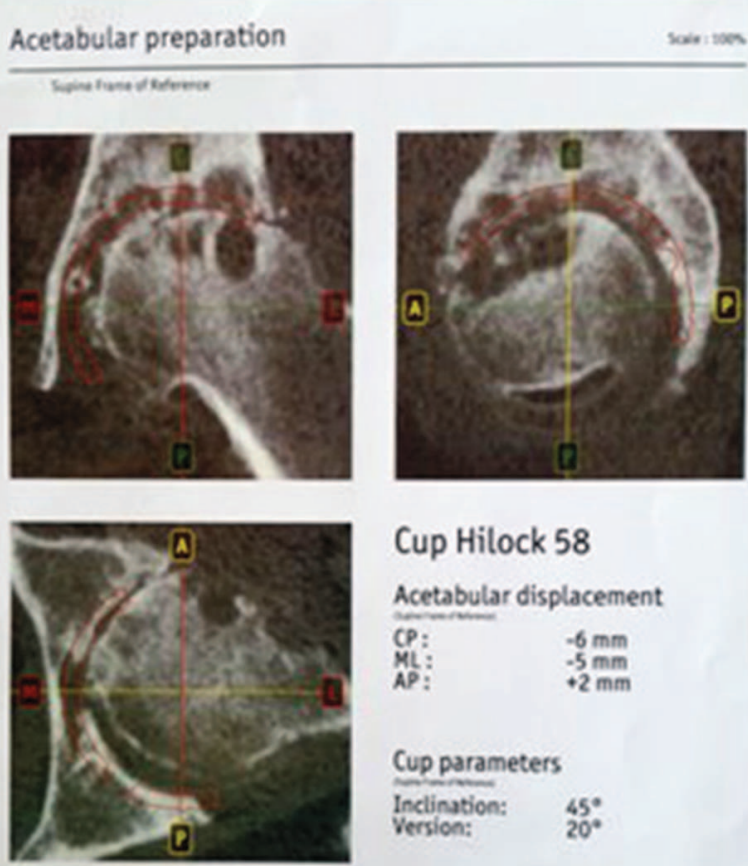

\section{Cup Hilock 58}

Acetabular displacement

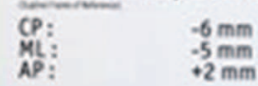

Cup parameters

Inclination: Version:

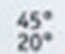

Remarks

Coosidention

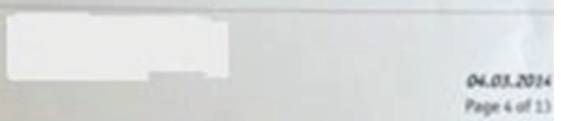

Fig. 9. Cup planning

The postoperative rehabilitation protocol includes immediate partial weight bearing in the first 3 weeks, depending on the patient's tolerance. Standard antithrombotic treatment with low-molecular heparin is performed for 35-40 days.

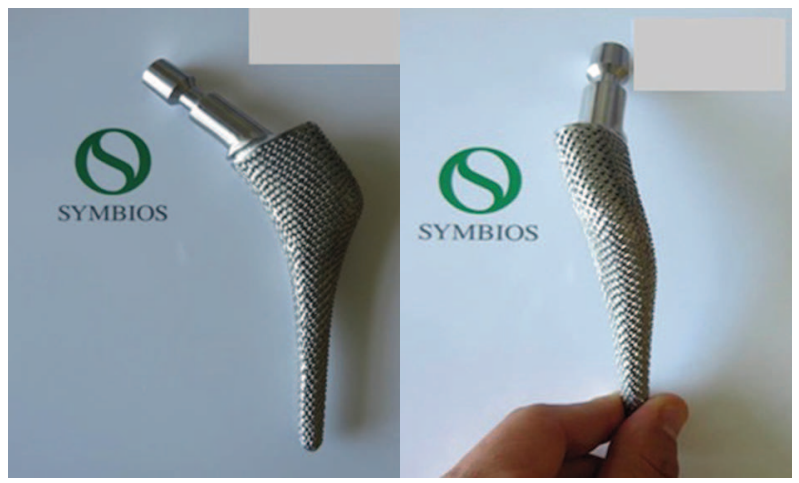

Fig. 10. Customized rasp (aggressive) used for impaction of spongious bone in the metaphyseal area. The custom stem has the same size and form 
All patients had a smooth postoperative period. We found no late complications. The average HarrisHips Score (HHS) was 97 (50-100) (Table 1). In 85\% of the patients, we had regular physical exercises with full ROM on the last follow-up visit. There were no pain or ROM limitations.

Table 1. Preoperative and postoperative HHS

\begin{tabular}{l|cc} 
Harris Hip Score & Preoperative & Postoperative \\
General & $41.9(25-88)$ & $97(50-100)$ \\
Pain & $18.2(10-44)$ & $42.6(20-44)$ \\
Walking & $9(2-11)$ & $31.7(9-33)$ \\
Activity & $7.6(2-14)$ & $13.5(6-14)$ \\
Deformity & $4.3(0-4)$ & $4(3-4)$ \\
ROM & $2.8(0-5)$ & $4.8(2-5)$ \\
\hline
\end{tabular}

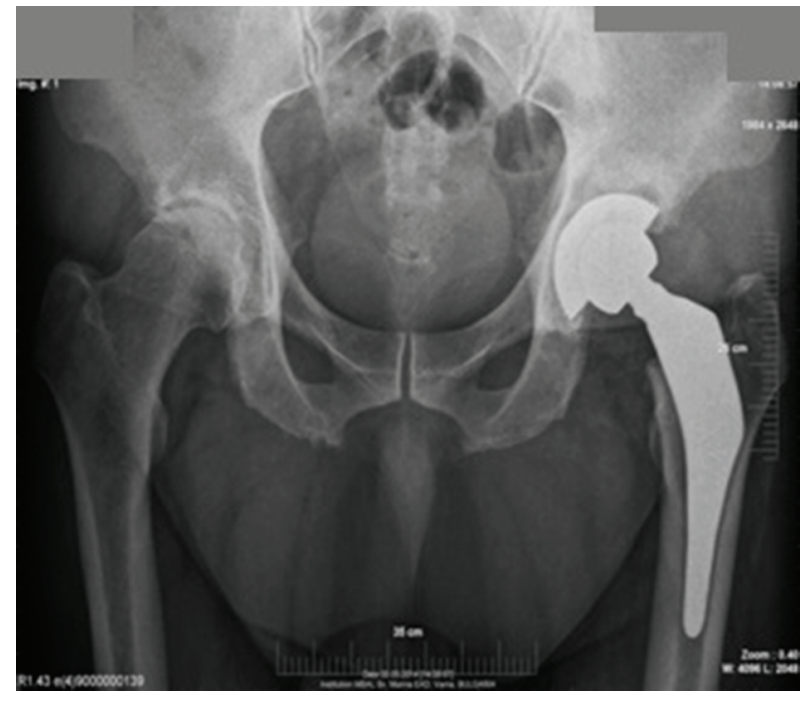

Fig. 11. Post-operative custom hip arthroplasty

\section{DISCUSSION}

THA is a challenge, when performed on young patients and those with anatomical deformities due to OA, dysplasia or surgical procedures (2).These cases present with an increased life expectancy, functional demand, ROM and implant longevity. Anatomical studies show that optimal prosthesis fit and fill in the metaphyseal zone and extramedullary adaptation is sometimes hard to achieve with conventional cementless stems $(10,14)$.

Stability of the cementless stems is secured with optimal fit and femoral canal preparation, but reaming stops when cortical bone resistance is reached. Due to that, the final stem size is therefore a compro- mise between the intramedullary anatomy and the bone density. An X-ray analysis of the femoral canal is insufficient, because it does not show these two parameters. The high accuracy of the $3 \mathrm{D}$ planning has been proved by Sugano et al. (11), who showed the inaccuracy of the X-ray analysis, when combining femoral anteversion with fixed external rotation over $15^{\circ}$. Such cases are recommended for 3D CT planning. 2D planning of the proximal stem fit to medial endosteal line is inaccurate, with sensitivity of $41 \%$ and specificity of $23 \%$. In 3D planning, the sensitivity is $93 \%$ and the specificity - $86 \%$.

The customized hip arthroplasty concept is from the 80s and the early results are worse than those with conventional stems. With the improvement of the diagnostic and manufacturing protocols,

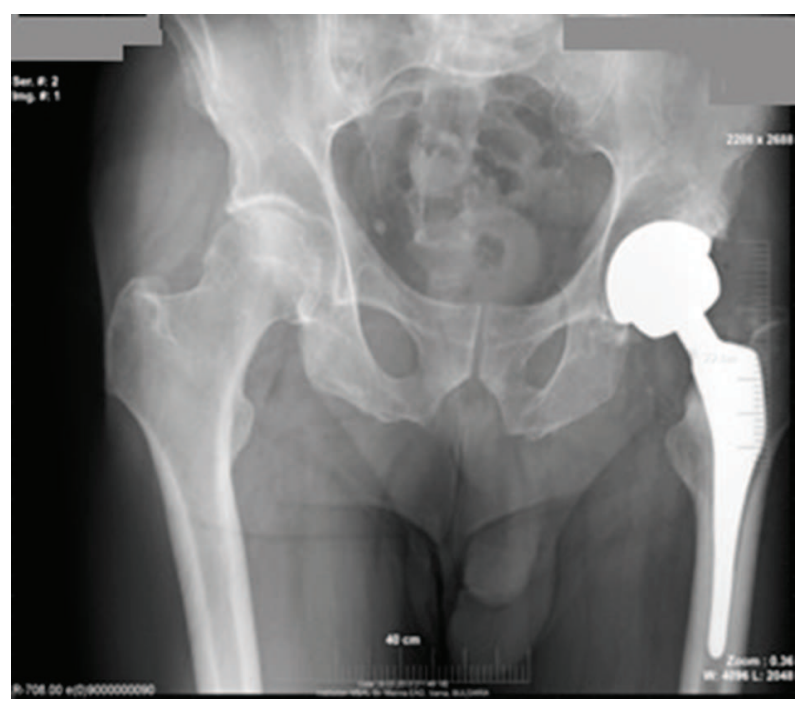

mean implant survival in the first decade is $100 \%$ in patients under 65 years with a substantial reduction of the clinical symptoms. Custom stem usage reduces the risk of early aseptic loosening, proximal stress shielding and femoral osteolysis $(7,9,15)$.

We believe that the greatest advantage of the customized stems is the $3 \mathrm{D}$ preoperative planning, responsible for stem manufacturing. It reproduces independently the extra- and intramedullary parameters, especially the cases with altered anatomy. Improved function, due to restored kinematics, component survival (optimal fit and fill) are keystones for a successful customized hip arthroplasty. 
Kalin Mihov, Maksim Zagorov, Svetoslav Dobrilov et al.

\section{CONCLUSION}

The custom stem has advantages especially when used in younger patients with anatomical alterations and high functional requirements. It achieves anatomical reconstruction and avoids intraoperative complications. These stems are a prerequisite for normal kinematics, low rate of bone restructuring and osteolysis, increased survival and low wear. They restore quickly, provide a high quality of life and physical activity.

\section{REFERENCES}

1. Asayama I, Chamnongkich S, Simpson KJ, Kinsey T, Mahoney O. Reconstruction hip joint position and abductor muscle strength after total hip arthroplasty. J Arthroplasty 2005;20:414-20

2. Bourne R, Rorabeck $\mathrm{CH}$. Soft tissue balancing: the hip. J Arthroplasty 2002;4:17-22.).

3. Capello WN, D’Antonio JA, Feinberg JR, Manley MT. Ten-year results with hydroxyapatite-coated total hip femoral components in patients less than fifty years old: a concise follow-up of a previous report. J Bone Joint Surg Am. 2003;85:885-889)

4. Charnley J. Low Friction Arthroplasty of the Hip. Theory and Practice. New York, NY: Springer; 1979

5. Collis DK. Long-term (twelve to eighteen-year) follow-up of cemented total hip replacements in patients who were less than fifty years old: a follow-up note. J Bone Joint Surg Am.1991;73:593-597

6. De Thomasson E, Mazel C, Guingand O, et al. Value of preoperative planning in total hip arthroplasty. Rev Chir Orthop 2002;88:229

7. Flecher X., Pearce O., Aubaniac JP, Argenson JN Custom Cementless Stem Improves Hip Function in Young Patients at 15-year Followup Clin Orthop Relat Res 2009

8. Flecher X., Pearce O., Aubaniac JP, Argenson JN Three-dimensional custom-designed cementless femoral stem for osteoarthritis secondary to congenital dislocation of the hip J Bone Joint Surg $[\mathrm{Br}]$ 2007;89-B:1586-91

9. Husmann O, Rubin PJ, Leyvraz PF, de Roguin B, Argenson JN.Three-dimensional morphology of the proximal femur. J Arthroplasty. 1997;12:444-450.)

10. Kim SY, Kyung HS, Ihn JC, Cho MR, Koo KH, Kim CY. Cementless Metasul metal-on-metal total hip arthroplasty in patients less than fifty years old. J Bone Joint SurgAm. 2004;86:2475-2481.

11. LaPorte DM, Mont MA, Hungerford DS. Proximally porouscoated ingrowth prostheses: limits of use. Orthopedics. 1999;22:1154-1160; quiz 1161-1162.

12. Massin $P$, Geais $L$, Astoin E, Simondi M, Lavaste F. The anatomic basis for the concept of lateralized femoral stems: a frontal plane radiographic study of the proximal femur. J Arthroplasty. 2000;15:93-101

13. McAuley JP, Szuszczewicz ES, Young A, Engh CA Sr. Total hip arthroplasty in patients 50 years and younger. Clin Orthop Relat Res. 2004;418:119-125

14. Nourbash PS, Paprosky WG. Cementless femoral designing concerns: rationale for extensive porous coating. Clin Orthop Relat Res. 1998;355:189-199.)

15. Rubin PJ, Leyvraz PF, Aubaniac JM, Argenson JN, Esteve P, de Roguin B. The morphology of the proximal femur: a three-dimensional radiographic analysis. J Bone Joint Surg Br. 1992;74:28-32

16. Sariali E., Mouttet A., Pasquier G., Durante E. Three-Dimensional Hip Anatomy in Osteoarthritis. Analysis of the Femoral Offset The Journal of Arthroplasty 2008

17. Sugano N, Ohzono K, Nishii T, et al. Computed tomography based computer preoperative planning for total hip arthroplasty. Comput Aided Surg 1998;3:320-4.) 